\title{
Carta abierta: acerca del mundo, los mundos y el papel de la filosofía*
}

\author{
Olimpia Lombardi**
}

\begin{abstract}
Resumen
Esta carta abierta es el resultado del intenso intercambio epistolar que he mantenido con el Prof. Torretti durante muchos años, y a través del cual me he enriquecido ampliamente en el pensar filosófico. Aquí señalo nuestros acuerdos en la adopción de una perspectiva de inspiración kantiana, y en el reconocimiento del papel que juega la dimensión pragmática en la ciencia. No obstante, nos distanciamos en cuanto al peso que le adjudicamos al realismo en nuestras posturas. Estas discusiones con el Prof. Torretti me permitieron explicitar mi modo de vivir la filosofía como praxis filosófica, dirigida a enfrentarme con ciertos aspectos del modo en que actualmente se hace ciencia.

Palabras clave: Realismo pluralista, mundo de la vida cotidiana, dominios ontológicos en ciencia, esquema conceptual, modelos, praxis filosófica.
\end{abstract}

\section{Open Letter: About the World, the Worlds and the Role of Philosophy}

\begin{abstract}
This open letter is the result of the intense epistolary exchange I had with Prof. Torretti for many years, which has highly enriched my philosophical thought. Here I point out our agreement in adopting a Kantian perspective, and in the acknowledgement of the role played by the pragmatic dimension in science. However, we drift apart regarding the weight assigned to realism in our positions. These discussions with

\footnotetext{
* Recibido: junio 2016. Aceptado: septiembre 2016.

** Universidad de Buenos Aires / Consejo Nacional de Investigaciones Científicas y Técnicas. Email: olimpiafilo@fibertel.com.ar
} 
Prof. Torretti led me to make explicit the way in which I live philosophy: as a philosophical praxis directed to struggle against certain aspects of contemporary science.

Keywords: Pluralist realism, the world of everyday life, ontological domains in science, conceptual scheme, models, philosophical praxis.

\section{Introducción}

No es fácil participar de un homenaje al Profesor Roberto Torretti. Por un lado, la responsabilidad me abruma un poco. Pero, por el otro, son tantas las cosas que tengo para decir que es difícil decidir cómo empezar y cómo organizarlas. Y es que desde hace muchos años vengo manteniendo con él un extenso intercambio de ideas, sobre todo por vía epistolar, intercambio que no sólo me ha enseñado muchísimo, sino que me ha abierto la mente para pensar desde nuevas y originales perspectivas. Por eso guardo celosamente en mi computadora (y en mis back-up's) los múltiples e-mails intercambiados, así como sus documentos en pdf llenos de globitos amarillos con observaciones que muchas veces valen más que un -buen- libro de filosofía.

Así me encontraba, entonces, enfrentándome al síndrome de la hoja en blanco, agravado por la responsabilidad de la tarea, cuando tuve una idea. En lugar de escribir un artículo tradicional, que nunca lograría hacer justicia al homenajeado, decidí escribir una carta que recogiera, de algún modo, nuestras discusiones epistolares. En una suerte de diálogo implícito, entonces, intentaré expresar mi postura frente a los desafíos que la potencia del pensamiento de Torretti fue imponiendo a mis ideas originales. Trataré de mostrar cómo, sobre un fondo de importantes acuerdos filosóficos, se fueron filtrando desacuerdos que me obligaron a pensar y repensar cuestiones centrales, desde la noción de mundo hasta el papel actual de la filosofía y el sentido de mi propia actividad filosófica.

Claro está que no voy a incluir otras citas más que las que provienen de los escritos publicados por el Profesor Torretti. Por lo tanto, en muchos casos responderé siguiendo mi propia interpretación de sus comentarios. Espero no distorsionar demasiado su pensamiento. De todos modos, incluso mediando involuntarias distorsiones, lo cierto es que mis intercambios con el Profesor Torretti han sido y continúan siendo un ingrediente central de mi crecimiento filosófico. 


\section{Carta abierta}

Querido Profesor Torretti:

Muchas gracias por sus comentarios acerca de nuestro libro': Ana Rosa y yo nos sentimos muy honradas por sus opiniones positivas y nos pone muy contentas comprobar los puntos de contacto entre nuestras posiciones.

\section{Encuentros relativistas y pragmáticos}

Creo que coincidimos en adoptar una perspectiva de inspiración kantiana ${ }^{2}$, que rechaza el compromiso del realismo metafísico con un mundo "ya hecho", conformado por objetos autosubsistentes y autoidentificantes, realismo que supone, además, la posibilidad de conocer dicho mundo tal como es en sí mismo, aunque sólo sea de manera aproximada. Como usted bien dice: "Los realistas cientificos creen que la realidad está bien definida de una vez por todas, independientemente de la acción humana y del pensamiento humano, de una manera que puede articularse adecuadamente en el discurso humano. Creen también que el propósito primordial de la ciencia es desarrollar justamente ese género de discurso que articula adecuadamente la realidad -que, como decía Platón, la "corta en sus coyunturas»- y que la ciencia moderna esencialmente está logrando ese propósito"3. Esta forma de realismo viene desmentida por la propia actividad científica. No sólo las propiedades y el comportamiento de las entidades estudiadas por la ciencia dependen del marco conceptual implícito en una teoría, sino también la propia existencia de esas entidades. Tan dependiente es la respuesta a '¿cómo es lo que hay?' como lo es la respuesta a ‘¿qué es lo que hay?'.

A diferencia del empirismo, que privilegia el ámbito de lo observable, el realismo que, siguiendo a Putnam ${ }^{4}$, denominamos 'internalista' adopta una posición "democrática" respecto de cualquier tipo de entidades: todo objeto, en tanto objeto de conocimiento, está conceptualmente constituido, incluyendo las entidades más directamente observables. Estoy segura de que usted concuerda en que lo observable también depende de la teoría. En otras

\footnotetext{
${ }^{1}$ Olimpia Lombardi y Ana Rosa Pérez Ransanz, Los Múltiples Mundos de la Ciencia, 2012.

2 Roberto Torretti, "Conocimiento discursivo", 2005; "Objectivity: a Kantian perspective”, 2008.

3 "Scientific realism and scientific practice", 2000. V.e. "El realismo científico y la ciencia como es", 2007 , p. 81.

${ }^{4}$ Hilary Putnam, Reason, Truth and History, 1981.
} 
palabras, la posesión de un marco conceptual, junto con los compromisos ontológicos que conlleva, son condición de posibilidad de la contrastación empirica.

Si bien un marco conceptual no es algo pasible de ser verdadero o falso, no cualquier marco es igualmente "bueno". Aquí nos hacemos eco del énfasis que usted siempre ha puesto en la praxis científica. En efecto, en su devenir histórico, la ciencia desarrolla una gran diversidad de prácticas, tanto teóricas como experimentales, a través de las cuales no sólo se consolida una teoría exitosa, sino también el marco conceptual sobre el que la teoría se asienta. Es ese marco conceptual el que interviene en la constitución del dominio al cual la teoría refiere. Se recupera así la intuición básica acerca del papel primordial que tiene la acción humana en la formulación, evaluación y estabilización de nuestras creencias sobre el mundo, y en particular de las teorías científicas.

El rechazo al realismo metafísico no se restringe a las ciencias naturales, sino que también alcanza al conocimiento histórico. Los enunciados formulados gramaticalmente en tiempo pasado resultan verdaderos o falsos en el esquema conceptual presente: el pasado existe única y exclusivamente como pasado del presente. $\mathrm{O}$ como usted mismo lo expresa de manera insuperable: "todo juicio se afinca en las vivencias, conceptos y valoraciones actuales -lo cual, por cierto, conferiría al presente una autoridad tiránica insoportable si no fuese que infalible e incesantemente se convierte en pasado".

Este realismo internalista y pragmático desemboca de manera natural en una postura anti-reduccionista cuando se consideran las relaciones entre teorías científicas y entre los dominios a los cuales éstas refieren. El supuesto metafísico de que existe un nivel ontológico fundamental y privilegiado no encuentra sustento en el análisis diacrónico ni el análisis sincrónico de las teorías científicas. La temperatura de un gas no es la energía cinética media de sus moléculas: la temperatura es aquello que, en el marco de la termodinámica, se mide con termómetros, que mantiene ciertas relaciones con la presión y el volumen, que se uniformiza siguiendo una ley definida, etc.; y ninguno de estos hechos requiere de una ontología subyacente para constatarse y establecerse como realmente existente. Supongo que usted coincidirá en que renunciar al reduccionismo no implica renunciar por completo al ideal de unificación en ciencias, si bien bajo una idea de unificación más flexible; una idea que se inscribe en la perspectiva abierta por Otto Neurath, cuando

\footnotetext{
5 "Inventar para entender", 2012.
} 
argumenta que la ciencia no se orienta hacia un sistema único, sino que procede mediante sistematizaciones locales, por lo cual tiene un carácter plural y siempre incompleto.

\section{Disensos terminológicos (¿sólo terminológicos?)}

Hasta aquí he comentado las coincidencias. Pero también agradezco profundamente sus críticas, porque me han hecho repensar cuestiones que, irreflexivamente, me parecían claras y no problemáticas.

En primer lugar, usted critica el uso que hacemos en nuestro libro de la palabra 'ontología' para referirnos a aquello a lo cual una teoría refiere. Considera que el uso es abusivo no sólo por la cantidad de veces que aparece en el texto, sino también porque es un término que originalmente significa "estudio del ente o de los entes". Desde mis primeros escritos sobre el tema usé ese término porque creía que con él desalentaría las lecturas meramente epistémicas de los realistas en su búsqueda de la "verdadera" realidad. Pero es cierto que, como usted sugiere, podría haber hablado de una realidad constituida o relativa a una teoría y su marco conceptual, pero insistiendo en que es la única realidad objeto de conocimiento. También podría usar la expresión 'dominio óntico', que tal vez le resulte más ilustrativa por sus reminiscencias matemáticas.

Otra expresión que veo no le ha gustado es 'esquema conceptual', en el sentido de sistema de conceptos y/o categorías. Y creo que no le ha gustado porque parece referir a un marco completo y cerrado. Por eso me propone 'tinglado conceptual', ya que es algo que se arma y desarma y se utiliza frecuentemente a medio armar; y que también puede desecharse después de usado, como la escalera de Wittgenstein. Me gusta la propuesta, si bien temo que habrá un número de filósofos hispanoparlantes que no sepan exactamente qué es un tinglado.

Tal vez lo que a usted le molesta de los términos 'ontología' y 'esquema conceptual' son sus "resonancias" de universalidad. Pero en nuestro caso se usan en un sentido "local", tanto desde un punto de vista histórico, como en un mismo momento del desarrollo de la ciencia. Por ejemplo, en nuestros días conviven múltiples dominios ónticos (ontologías) y, correspondientemente, múltiples tinglados (esquemas) conceptuales, incluso en el seno de la propia física: hay un dominio mecánico-clásico, un dominio relativista general, un dominio termodinámico, habría un dominio mecánico-cuántico si lográramos ponernos de acuerdo con su interpretación, etc. Los físicos que se ocupan de 
las diferentes teorías trabajan con dominios ónticos diferentes, si bien eso no significa que tales dominios no tengan nada en común: algo deben compartir la mecánica clásica y la relatividad general para que se puedan, al menos en principio, predecir los mismos hechos empíricos en ambas.

Creo que es en este sentido local del trabajo científico que puede decirse que, por ejemplo, los físicos que trabajan en teorías diferentes "viven en mundos distintos". En nuestro libro nosotras usamos la palabra 'mundo' de un modo que permite la multiplicidad de mundos (el mundo aristotélico, el mundo de la mecánica clásica, el mundo de la termodinámica, el mundo de nuestro sentido común, etc.). No obstante, ya me parece escuchar su exclamación: "Pero Olimpia, ¡el mundo es siempre global!", en consonancia con su conocido disgusto respecto de la expresión kuhniana. Quien nos escuchara podría pensar que se trata de un disenso puramente terminológico, que podría subsanarse si encontráramos un término mejor que 'mundo' o si, sencillamente, agregáramos a la palabra los subíndices 'G' y 'L' ('global' y 'local') para regocijo de los filósofos analíticos. Pero me parece que hay algo más, que hay un desacuerdo conceptual subyacente a la cuestión terminológica: veamos si me equivoco.

\section{Desencuentros en el mundo de la vida cotidiana}

Para nosotras, todos los dominios ontológicos, tanto los de la vida cotidiana como los de la ciencia, son dependientes de algún tinglado conceptual $\mathrm{y}$, en principio, poseen el mismo rango ontológico; no existe un dominio privilegiado, un estrato fundante o fundamental respecto de los restantes. Yo sé que, desde su fuerte antifundacionismo, en principio usted simpatizará con esta postura. Sin embargo, ha sido muy claro al afirmar que la vida del intelecto, y aun la de la práctica de laboratorio, presuponen la vida cotidiana integral, donde nos hacemos problema por nuestros hijos (si sabré de eso...), sacamos fotos a nuestros nietos, y comemos sandwiches de jamón y palta (aguacate, para Ana Rosa). Como usted explica claramente en Creative Understanding: "Ninguna teoría fisica reivindica una comprensión global de la realidad. El fisico que sustituye una teoría por otra sigue viviendo en el mismo vecindario, trabajando en el mismo instituto, conduciendo cada día por el mismo viejo camino entre ellos, de un lado al otro, mientras reflexiona acerca de la política de su país, o los humores de sus hijos adolescentes, o las fallas de su automóvil, a la luz de los mismos conceptos sociales, morales y de bajo nivel mecánico que usaba antes. El dictum de Kuhn de que "después de una revolución los científicos viven en mundos diferentes» es una pieza de 
retórica vacía, o bien un malentendido acerca de la naturaleza y los alcances

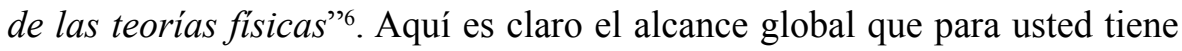
la palabra 'mundo'. Pero no sólo eso: creo que lo que se manifiesta es que, entre aquellos dominios que nosotras consideramos con el mismo estatuto ontológico, para usted hay uno privilegiado, que es el de la vida cotidiana. Así como para algunos el lenguaje natural es el nivel lingüístico último, el metalenguaje de todos los lenguajes, el mundo de la vida cotidiana brinda para usted el escenario común donde despliegan sus papeles los diferentes dominios de la ciencia.

Para nosotras, en cambio, nuestro mundo cotidiano es un mundo, pero no el mundo. Es un mundo bastante distinto del aquél del pequeño pueblo de Sicilia donde nació y vivió hasta hace poco mi abuela materna. Para sus habitantes más ancianos, muchos completamente analfabetos y con creencias místicas muy fuertes, el parentesco, la muerte, y hasta el espacio y el tiempo son completamente diferentes de los nuestros: todos los que emigraron viven en un lugar indeterminado que se llama "l'America", no importa si se instalaron en Chicago o en Buenos Aires; la primera vez que estuve allí, la gente me confundía con mi madre, quien había dejado el pueblo con la edad que yo tenía entonces pero 40 años antes... Y si estas diferencias se pueden dar entre culturas occidentales y actuales, tengo que admitir que el quiebre puede ser mucho más profundo aún entre comunidades más alejadas culturalmente y/o históricamente. El monje benedictino del siglo XI no hacía casi nada de lo que hacemos hoy, y la mayoría de las cosas que él hacía ya no las hacemos nosotros; su realidad estaba poblada de entidades inobservables que no existen en nuestra realidad, y viceversa. Imaginémonos transportados a un monasterio cluniacense; supongo que ni siquiera físicamente podríamos resistir: nuestros propios estómagos no resistirían la comida, ni nuestros huesos el frío, ni nuestras narices los olores. Esto es lo que nos lleva a admitir la diversidad de realidades constituidas por los tinglados conceptuales y las prácticas de cada comunidad no sólo en el ámbito de la ciencia, sino en todo ámbito humano.

En otras palabras: el mismo argumento por el cual afirmamos que hay diferentes dominios ónticos en la ciencia, tanto diacrónicamente (el dominio aristotélico, el newtoniano, etc.) como sincrónicamente (el dominio de la mecánica clásica, del electromagnetismo, etc.), nos obliga a admitir la multiplicidad de realidades constituidas no-científicas. De ahí se desprenden nuestras afirmaciones acerca de la "democracia" entre los diferentes mundos, no sólo

${ }^{6}$ Creative Understanding, 1990, p. 79. 
los de la ciencia sino también los del sentido común. En este sentido somos relativistas, y así nos ven con espanto muchos filósofos de la ciencia tradicionales, más horrorizados aún aquéllos que creen que es la ciencia la que descubre la "verdadera" realidad.

\section{Desencuentros en el ámbito de la ciencia}

Si nuestros primeros desencuentros se manifiestan respecto del mundo cotidiano, no es de sorprender que se extiendan al ámbito de la ciencia. Usted considera que nosotras no nos hemos deshecho suficientemente del bagaje cartesiano, y quizás hartmanniano, con el que nos cargaron en la escuela. Y esto es así porque, a su modo de ver, ni la ciencia ni la filosofía crítica están en el negocio de cultivar concepciones del mundo: "Llego a esta conclusión cuando veo la forma cómo teorías discrepantes se aplican conjuntamente en la consideración de ciertos fenómenos y en la solución de ciertos problemas" . Como ejemplos nos ofrece los cálculos de la trayectoria de Mercurio, que utilizan simultáneamente mecánica clásica y relatividad especial, y el estudio de Hawking sobre la evaporación de agujeros negros, que "combina descaradamente teorías incompatibles" $"$ como la relatividad general y la mecánica cuántica.

Sin duda, los ejemplos están inmejorablemente elegidos: las cosas sucedieron exactamente como usted dice. Sin embargo, me parece que disentimos respecto del valor que les adjudicamos. Para usted, estos casos son constitutivos de la propia actividad científica, que no se ocupa de brindar una visión consistente de lo real, sino de describir fenómenos y resolver problemas de un modo eficiente. Pero, creo, con ello se pierde el ingrediente central del realismo, para virar hacia una concepción más instrumental de la ciencia. La perspectiva realista que defiendo, en cambio, no puede integrar la inconsistencia como algo natural o propio de la ciencia. Considero que el realismo exige, al menos, admitir que la actividad teórica y práctica de la ciencia está destinada a dar cuenta de algún dominio de lo real, y para ello la coherencia es un elemento irrenunciable. Las situaciones en las cuales teorías incompatibles se combinan descaradamente existen, sin duda, pero no suelen ser vistas por los científicos como constitutivas de su actividad, sino como limitaciones provisorias que se disipan con el aumento de la potencia de cálculo o que se resolverán con nuevos desarrollos teóricos.

\footnotetext{
7 "El realismo científico y la ciencia como es", p. 81.

${ }^{8}$ Ibid. p. 91
} 
En definitiva, para el realismo al que adhiero, la actividad científica construye modelos que integran teorías incompatibles cuando no se cuenta con algo mejor; pero siempre se preferirán las descripciones basadas en una manera coherente de concebir lo real. Claro que esto no significa que el objetivo de la ciencia sea brindar una cosmovisión global y omniabarcante. Desde mi perspectiva pluralista, la práctica científica nos ofrece diferentes visiones no integrables en una cosmovisión única; pero cada una de ellas es consistente en sí misma en la medida en que refiere a un dominio óntico: en ello reside el ingrediente realista de mi posición.

\section{Esquemas, teorías y modelos}

Esta diferencia entre una aproximación más instrumental a la ciencia y una más realista adquiere una nueva manifestación en el modo en que concebimos los modelos en ciencias.

Cuando discutíamos acerca de la noción de mundo, usted señaló que los modelos de masas puntuales para representar el sistema solar formaban parte del dominio óntico de la mecánica celeste del siglo XIX, poblada precisamente por masas puntuales y fuerzas gravitatorias entre ellas. Sobre esta base, usted razonablemente concluye que 'realidad constituida' significa algo distinto de 'mundo': en el siglo XIX nadie vivía en un "mundo" de masas puntuales. Sin embargo, si no comprendí mal, esta conclusión requiere suponer que los modelos son o intervienen en la constitución de los diferentes dominios ónti$\cos$ de la ciencia. $\mathrm{Y}$ aquí nuestras posiciones volverían a divergir.

Para mí existe una diferencia esencial entre dominio óntico y modelo. El dominio óntico es esa realidad constituida por el tinglado conceptual que subyace a una cierta teoría; pero para aplicar la teoría el científico se vale de un modelo, que es un sistema conceptualmente construido que media entre dicha teoría y la realidad constituida. El científico tenderá a usar el modelo más sencillo hasta que ya no le sirva. Por ejemplo, tal vez se pueda modelizar una pelota de futbol como una masa puntual para explicar un penalty, pero tal modelo no sirve para explicar un gol olímpico, que exige concebir la pelota como un cuerpo rígido girando en su desplazamiento a través del aire. Pero no diría que ambos modelos son distintos dominios ónticos: ambos se usan para representar una misma realidad constituida, como lo es la de la mecánica clásica, "habitada" por cuerpos que chocan y se deforman en mayor o menor medida, cuerpos que orbitan unos respecto de otros, materiales que pueden transmitir ondas transversales, fluidos donde pueden producirse ondas longitudinales, etc. Pero en esta realidad no existen las ondas electromagnéticas, 
y cuando Maxwell intentó demostrar que tales ondas también "habitaban" el dominio de la mecánica clásica, ya que no eran más que vibraciones mecánicas del éter, no le fue nada bien (si bien no es necesario que siempre sea así). En el ámbito mecánico-clásico, puedo modelizar el gas contenido en un recipiente como un gas de Lorentz (partículas puntuales que, por tanto, sólo chocan con las paredes del recipiente pero no entre sí), o puedo ir complejizando el modelo considerando las partículas con un cierto volumen para que choquen elásticamente entre sí, o puedo adjudicarles un coeficiente para que su choque sea plástico y se deformen, etc.; pero en todos estos casos se trata de modelos que median entre mi teoría (mecánica clásica) y el dominio óntico mecánico-clásico, donde no existe la temperatura, que sí habita en el dominio de la termodinámica9 9 .

Volviendo a su ejemplo de la mecánica celeste del siglo XIX, yo diría que se trabajaba en la realidad constituida de la mecánica clásica (donde no existen fuerzas eléctricas ni magnéticas, ni ondas electromagnéticas, ni calor ni temperatura, ni reacciones químicas, etc.), utilizando un cierto tipo de modelos, que trataba los planetas como masas puntuales. Pero si se le preguntara a un físico de la segunda mitad del siglo XIX acerca de lo existente, no creo que respondiera que en la realidad había masas puntuales. No obstante, la mayoría de los físicos de la época seguramente habría negado la existencia del calor y de las ondas electromagnéticas como entidades diferentes del mero movimiento de partículas o de medios mecánicos.

Son, entonces, los esquemas o tinglados conceptuales los que intervienen en la constitución de los dominios ónticos, no los modelos. Los modelos intervienen en la aplicación de una teoría, mediando entre la propia teoría y la realidad previamente constituida, donde 'previamente' debe entenderse en un sentido lógico y no temporal. En efecto, es claro que, desde un punto de vista histórico-genético, en el proceso de consolidación de una teoría con su tinglado conceptual, en general no se pueden diferenciar como momentos distintos la constitución de la realidad, la formulación de una teoría y la utilización de cierto o ciertos modelos que funcionan como los referentes paradigmáticos de la teoría. Pero esto no cancela la diferencia conceptual entre los tres niveles: el tinglado conceptual es el que interviene en la constitución del dominio óntico, la teoría se refiere a dicho dominio -y puede haber muchas teorías, incluso contradictorias, que hablen de la misma realidad-, y

\footnotetext{
9 Desarrollé esta visión de los modelos científicos en algunos trabajos como "La noción de modelo en ciencias", 1998, "Los modelos como mediadores entre teoría y realidad", 2010 y "Acerca del modo en que la ciencia refiere a lo real: el papel de los modelos", 2012 (en colaboración).
} 
los modelos son sistemas conceptualmente construidos que se utilizan para ciertos fines particulares. Por lo tanto, siempre hay múltiples modelos en la aplicación de una misma teoría científica, pero -en caso de tener pretensiones representacionales- todos ellos representan parcialmente la misma realidad constituida: no pueden representar aquello que en esa realidad no existe (no hay modelo de ondas electromagnéticas viajando en el vacío en la realidad constituida por la mecánica clásica). No obstante, los científicos no creen que aquello a lo cual los modelos refieren sea la realidad (constituida o no), sino que consideran que los modelos refieren a construcciones conceptuales que "viven" en sus propias cabezas y en sus propias prácticas. Dicho en palabras sencillas: en la realidad constituida por el tinglado conceptual de la mecánica clásica existen los planetas del sistema solar, pero no las masas puntuales del modelo de masas puntuales, aun si sólo pudiera aplicarse la teoría al sistema solar a través del modelo de masas puntuales.

Todo lo antedicho supone que los modelos de algún modo representan lo real. Pero actualmente hay una tendencia a considerar que la representatividad no es condición para que un modelo sea tal: diversos autores ponen ejemplos de modelos que no pueden resultar representativos en la medida en que contradicen la teoría sobre la que parcialmente se basan ${ }^{10} \mathrm{o}$ integran teorías incompatibles ${ }^{11}$. Este sería el caso de los modelos de evaporación de agujeros negros propuestos por Hawking que usted menciona. De este modo, incluso un realista puede aceptar que los modelos son puramente instrumentales, si bien suponiendo que las teorías intentan describir algún dominio de lo real.

Desde esta perspectiva, concibo tres formas posibles de cambio científico, que ordenaré desde la más a la menos conservadora. Consideremos el resultado negativo del experimento de Michelson-Morley:

- La primera estrategia, y la más conservadora, es mantener la teoría (y con ello, la realidad constituida) y cambiar el modelo. En el caso considerado, se intentó modelizar el éter de modo que pudiera producir un "viento" capaz de dar cuenta del resultado negativo.

- La segunda estrategia es menos conservadora. Si el primer camino no funciona porque no se encuentra un modelo tal que, al aplicarle la teoría, brinde resultados empíricamente adecuados, el científico opta por intentar el cambio de teoría, pero preservando el mismo tinglado de

\footnotetext{
${ }^{10}$ Ver, e.g., Mauricio Suárez y Nancy Cartwright, “Theories: tools versus models”, 2008.

${ }^{11}$ Ver, e.g., Hernán Accorinti y Juan Camilo Martínez González, “Acerca de la independencia de los modelos respecto de las teorías: un caso de la química cuántica”, 2016.
} 
categorías y conceptos, o sea, manteniéndose en el marco del mismo dominio óntico. Esto es factible porque se pueden formular diferentes teorías sobre la base de un mismo tinglado conceptual, esto es, referidas a una misma realidad constituida. Éste sería un cambio teórico no revolucionario. En nuestro caso, ésta fue la alternativa de Lorentz y FitzGerald.

- La tercera estrategia es la menos conservadora, es la "revolucionaria", y consiste en cambiar el propio tinglado de categorías y conceptos y, con él, la realidad constituida. En el ejemplo histórico considerado, ésta es la opción de Einstein. Esto no significa que ambos tinglados no tienen nada en común (no significa que ambas realidades no comparten nada), porque si así fuera no podríamos siquiera entender por qué se admite que, en algunos casos, teorías que refieren a dominios ónticos diferentes pretenden resolver los mismos problemas. Pero esto no cancela el hecho de que dichas teorías nos hablan de realidades constituidas diferentes.

En definitiva, desde mi punto de vista existe una diferencia central entre modelo, teoría y tinglado conceptual-realidad constituida. Un sustrato categorial-conceptual, en tanto interviene en la constitución del dominio óntico, es mucho más estable que las diferentes teorías que pueden formularse sobre su base. A su vez, una teoría científica es mucho más general y estable que los múltiples modelos que se usan en su aplicación: los modelos median en la relación entre teoría y realidad constituida; tienen un papel instrumental ya que se los utiliza para fines específicos y son los primeros elementos que los científicos están dispuestos a abandonar en caso de desajustes empíricos. La distinción entre estos tres conceptos es muy importante para mí, por razones que quedarán claras más abajo.

\section{6. ¿Para qué la filosofía?}

Hace ya varios años, participé en $\operatorname{SADAF}^{12}$ de un homenaje a Eduardo Rabossi un tiempo después de su inesperado fallecimiento. Uno de los oradores, español y filósofo analítico, presentó una fuerte reflexión acerca del "para qué" del trabajo filosófico, en un ejercicio en el que asumía que la filosofía (principalmente la analítica) tiene un desarrollo endogámico con poca comunicación con el "afuera" de la propia actividad específica. La charla fue

\footnotetext{
12 Sociedad Argentina de Análisis Filosófico, Buenos Aires.
} 
bastante movilizadora para todos los presentes. Sin embargo, yo no me sentí particularmente afectada, porque tiendo a vivir la filosofía como praxis filosófica. Siempre digo que intento hacer, no filosofía de la ciencia, sino filosofía para la ciencia: creo que la reflexión filosófica puede ayudar a los científicos, no a convertirse en filósofos, sino a hacer mejor ciencia en la medida en que les permite reflexionar acerca de lo que hacen, y eso estimula el pensamiento crítico y la creatividad. Esto, por supuesto, se basa en mi convicción de que la actividad científica es una actividad humana valiosa que debemos promover. Es por ello que considero esencial para el filósofo de la ciencia el contacto con científicos, y siempre intento participar en cursos y charlas para estudiantes de grado y posgrado en ciencias.

El caso es que, desde que me enfrento a doctorandos y algunos jóvenes doctores en ciencias que asisten a mis cursos, me di cuenta de qué sucede con esta gente inmersa en el trabajo científico cotidiano. Casi en su totalidad, estos jóvenes fueron y siguen siendo educados en la idea de la "verdad verdadera"13, en la visión de una ciencia que corre el velo de la realidad para descubrirla tal como es de una vez y para siempre. Estos jóvenes (al menos los que pueden "liberarse" lo suficiente como para asistir a cursos o charlas de filosofía), sin embargo, se dan cuenta de que la práctica de su propia disciplina no responde a esa imagen ingenua, y así comienza el desarrollo de una crisis interna que muchos nunca podrán superar.

Es muy frecuente que en la facultad de ciencias o en conferencias a las que asisten jóvenes científicos aparezca algún sociólogo de la ciencia o filósofo posmoderno que sostiene que la ciencia no es más que mera negociación entre individuos que buscan su promoción en la carrera académica, o que todo es discurso y no hay nada detrás de las palabras. Y estos chicos, desilusionados de la "verdad verdadera", compran este discurso relativista del "todo vale lo mismo", que se da de patadas con su actividad científica cotidiana, en la cual pasan meses tratando de resolver una ecuación o tienen que ir a la facultad los fines de semana a alimentar a los organismos que estudian en el laboratorio. El resultado es pura esquizofrenia y frustración, que en algunos casos los afecta psicológicamente de una manera profunda: ya no saben para qué hacen lo que hacen.

En este marco, el relativismo no-salvaje que tratamos de delinear con Ana Rosa, basado en ciertas intuiciones kantianas, está teniendo una muy buena

\footnotetext{
${ }^{13}$ Ver "Ciencia provisoria", firmado por "Maestro Ciruela", quien finalmente se reconoció como el editor en jefe del órgano difusión de la Facultad de Ciencias Exactas y Naturales de la Universidad de Buenos Aires.
} 
recepción en el ámbito de la ciencia. Sin duda nuestro enfoque tiene muchas aspectos por discutir y pulir, pero ya actualmente está mostrando sus frutos en esto que llamo "praxis filosófica". Porque nuestro realismo pluralista les permite a los jóvenes científicos y proto-científicos descartar de una vez por todas la idea de la "verdad verdadera", sin que ese movimiento los conduzca a un relativismo "salvaje" que priva de todo sentido sus esfuerzos, tanto teóricos como experimentales. Pueden acomodar sus intuiciones relativistas (que, si bien borrosas, las tienen) en un contexto en el cual la actividad científica de "preguntar" a ese algo que está fuera de ellos mismos interviene en la construcción del conocimiento. Mi sueño, que seguro no veré, es que algún día esos jóvenes puedan llegar a posiciones institucionales que les permitan promover una actividad científica más rica y reflexiva que la que actualmente se desarrolla en las facultades de ciencias, al menos en la Argentina.

Ahora bien, hay una tercera posición, ortogonal a las de "la verdad verdadera" y del "todo vale lo mismo", que es la postura "superada" del instrumentalismo extremo: es la de aquellos científicos que miran a la filosofía con indulgencia en el mejor de los casos, y en el peor con desprecio. Ellos "saben" que la ciencia no requiere ninguna reflexión filosófica puesto que su único objetivo es la predicción y el control. Entonces, como para ellos todo lo científico es instrumental, es tan instrumento lo que yo llamo 'teoría' como lo que yo llamo 'modelo'. La actividad científica se reduce a construir modelos con fines exclusivamente prácticos.

Creo que era Nagel quien decía, acerca del instrumentalismo en relación con los llamados términos teóricos de la ciencia, que finalmente ser instrumentalista o realista es una cuestión puramente filosófica que no afecta al propio desarrollo científico. Sea que esté reconstruyendo bien o no esta idea, me permito disentir con ella. Creo que el instrumentalismo que lleva a pensar que todo es modelo, que cancela la diferencia entre teoría y modelo, le hace mucho mal a la propia ciencia, en particular a la física. Actualmente, una enorme proporción del trabajo de los físicos teóricos consiste en postular modelos, describirlos (matemáticamente las menos de las veces, mediante simulaciones numéricas por computadora casi siempre), y publicar un paper con los resultados numéricos obtenidos. En sí mismo esto no está mal, pero inquieta cuando se comprueba que es aquello en lo que se invierte la mayor parte del esfuerzo científico, bajo el supuesto de que eso es hacer física teórica: claro, cuando ya todo es modelo, la actividad teórica no es más que la actividad de hacer modelos. El resultado es una superpoblación de trabajos totalmente insignificantes, que no hacen más que repetir lo ya publicado con leves modificaciones. 
Esto lo he vivido en carne propia. Junto a Mario Castagnino (con quien trabajé hasta su retiro, un par de años atrás) y Sebastián Fortin (actualmente mi principal colaborador) formulamos un enfoque de la decoherencia cuántica más general que el ortodoxo, que convierte la decoherencia en un fenómeno relativo a la partición del sistema cuántico cerrado que se considere. Para ilustrar esta propuesta conceptual, Sebastián formuló un modelo y lo describió por computadora (ya que Castagnino y yo somos totalmente incapaces de hacerlo); con este material escribimos un artículo que enviamos a una muy buena revista internacional de física. El artículo fue rechazado con un referato de cinco líneas según el cual (i) todo lo que decíamos estaba mal, porque "se sabe" que la decoherencia es tal como sostiene el enfoque ortodoxo, pero (ii) el modelo es interesante. Después de pensar largamente sobre qué hacer, decidimos dejar el modelo solo, completamente "pelado", y expurgar el artículo de todo vestigio de pensamiento. Resultado: el paper fue aceptado de inmediato en la misma revista y sin una sola observación ${ }^{14}$. Como dicen Les Luthiers: "el que piensa, pierde".

En el marco de esta manera, a mi modo de ver nefasta, de hacer "física teórica", conozco a alguien que consiguió para su laboratorio un aparato que le permitió llevar adelante la siguiente estrategia durante años: formular un modelo, medir y publicar, modificar un poco el modelo, medir y publicar, y así siguiendo, como en una línea de montaje (ahora ya no lo hace porque es alto funcionario de la universidad). En el mismo marco, los físicos teóricos más "exitosos" son los que consiguen más estudiantes de doctorado, a quienes ponen a resolver modelos que luego publicarán juntos; de este modo, el físico exitoso se torna cada vez más exitoso y con ello aumenta el número de miembros de su ejército de resolvedores de modelos. Y así se inundan las revistas, que cada vez son más, de artículos carentes de dimensión teórica y producto de un trabajo totalmente rutinario. Los doctorandos en física, nuevamente, sufren las consecuencias de esta situación: los más reflexivos se frustran y, en muchos casos, abandonan el doctorado o, si bien acaban doctorándose, pierden el tren de la investigación en física o, en el mejor de los casos, quedan relegados en la carrera; paradójicamente, los que logran mantenerse y tienen éxito son los menos reflexivos, los que mejor se adecuan al trabajo rutinario y se convierten en los futuros físicos instrumentalistas "superados". Y todo esto justificado por el supuesto de que están haciendo física teórica, ya que no

\footnotetext{
14 "Suppression of decoherence in a generalization of the spin-bath model", 2010. La parte conceptual dio lugar al artículo "The problem of identifying the system and the environment in the phenomenon of decoherence", 2012, que utilizó los resultados del artículo anterior como ilustración, y que fue publicado en una compilación de artículos filosóficos de la European Philosophy of Science Association.
} 
hay diferencia entre teoría y modelo. Estoy convencida de que esta situación, que presencio diariamente, no le hace bien a la física. Y parte de mi praxis filosófica se dirige a denunciar esta situación, con todas las consecuencias que se siguen de ello.

\section{Coda}

Todo este largo discurso puede interpretarse como mostrando que nuestros disensos son muchos y profundos. Sin embargo, no es tan así: como usted sabe, coincidimos en muchos más aspectos de aquéllos en los que disentimos. Pero no es el caso de pasar el rato tirándonos flores, como si fuéramos políticos de un mismo partido después de las elecciones internas. La filosofía es disenso y discusión, en ello radica su esencia y su riqueza. Y es por ello que usted me ha ayudado tanto a ser mejor filósofa.

Con todo mi cariño y admiración.

Olimpia.

\section{Bibliografía}

Accorinti, H. y Martínez González, J. C. (2016). “Acerca de la independencia de los modelos respecto de las teorías: un caso de la química cuántica", Theoria, en prensa.

Cabrera, R. (2009). "Ciencia provisoria”, Exactamente (Facultad de Ciencias Exactas y Naturales de la Universidad de Buenos Aires), 44: 47.

Castagnino, M., Fortin, S. y Lombardi, O. (2010). "Suppression of decoherence in a generalization of the spin-bath model", Journal of Physics A: Mathematical and Theoretical, 43: 065304.

Córdoba, M. y Lombardi, O. (2012). "Acerca del modo en que la ciencia refiere a lo real: el papel de los modelos", pp. 93-102, en Z. Monroy Nasr, R. León-Sánchez y G. Álvarez Díaz de León (eds.), Enseñanza de la Ciencia. México: Universidad Nacional Autónoma de México.

Lombardi, O. (1998). "La noción de modelo en ciencias", Educación en Ciencias, 2: 5-13.

Lombardi, O. (2010). "Los modelos como mediadores entre teoría y realidad", pp. 83-94, en L. Galagovsky (ed.), Didáctica de las Ciencias Naturales. El Caso de los Modelos Cientificos. Buenos Aires: Editorial Lugar. 
Lombardi, O., Fortin, S. y Castagnino, M. (2012). "The problem of identifying the system and the environment in the phenomenon of decoherence", pp. 161-174, en H. W. de Regt, S. Hartmann y S. Okasha (eds.), EPSA Philosophy of Science: Amsterdam 2009. Berlin: Springer.

Lombardi, O. y Pérez Ransanz, A. R. (2012). Los Múltiples Mundos de la Ciencia. Un Realismo Pluralista y su Aplicación a la Filosofia de la Física. México: UNAM-Siglo XXI.

Putnam, H. (1981). Reason, Truth and History. Cambridge: Cambridge University Press.

SuÁrez, M. y Cartwright, N. (2008). "Theories: tools versus models", Studies in History and Philosophy of Modern Physics, 39: 62-81.

Torretti, R. (1990). Creative Understanding: Philosophical Reflections on Physics. Chicago: The University of Chicago Press.

TORRETTI, R. (2000). "Scientific realism and scientific practice", pp. 113-122, en E. Agazzi y M. Pauri (eds.), The Reality of the Unobservable: Observability, Unobservability and their Impact on the Issue of Scientific Realism. Dordrecht: Kluwer. V.e. (2007). "El realismo científico y la ciencia como es", pp. 75-98, en Escritos Filosóficos 1986-2006. Santiago de Chile: Universidad Diego Portales.

Torretti, R. (2005). “Conocimiento discursivo", Lección Inaugural dictada en la Universidad Autónoma de Barcelona, 28 de Abril de 2005, en ocasión de la investidura del autor como Doctor Honoris Causa de dicha Universidad.

TorretTi, R. (2008). “Objectivity: a Kantian perspective”, pp. 81-95, en M. Massimi (ed.), Kant and Philosophy of Science Today. Cambridge: Cambridge University Press.

TorretTI, R. (2012). "Inventar para entender", Lección Inaugural dictada en la Universidad Diego Portales, 27 de Marzo de 2012, Santiago de Chile. 
